\title{
A BRDF-BPDF database for the analysis of Earth target reflectances
}

\author{
Francois-Marie Breon and Fabienne Maignan \\ Laboratoire des Sciences du Climat et de l'Environnement (LSCE/IPSL), UMR CEA-CNRS-UVSQ, \\ University Paris-Saclay, Gif-sur-Yvette, 91191, France \\ Correspondence to: Francois-Marie Breon (breon@1sce.ipsl.fr)
}

Received: 29 August 2016 - Published in Earth Syst. Sci. Data Discuss.: 28 September 2016

Revised: 25 December 2016 - Accepted: 2 January 2017 - Published: 20 January 2017

\begin{abstract}
Land surface reflectance is not isotropic. It varies with the observation geometry that is defined by the sun, view zenith angles, and the relative azimuth. In addition, the reflectance is linearly polarized. The reflectance anisotropy is quantified by the bidirectional reflectance distribution function (BRDF), while its polarization properties are defined by the bidirectional polarization distribution function (BPDF). The POLDER radiometer that flew onboard the PARASOL microsatellite remains the only space instrument that measured numerous samples of the BRDF and BPDF of Earth targets.

Here, we describe a database of representative BRDFs and BPDFs derived from the POLDER measurements. From the huge number of data acquired by the spaceborne instrument over a period of 7 years, we selected a set of targets with high-quality observations. The selection aimed for a large number of observations, free of significant cloud or aerosol contamination, acquired in diverse observation geometries with a focus on the backscatter direction that shows the specific hot spot signature. The targets are sorted according to the 16-class International Geosphere-Biosphere Programme (IGBP) land cover classification system, and the target selection aims at a spatial representativeness within the class. The database thus provides a set of high-quality BRDF and BPDF samples that can be used to assess the typical variability of natural surface reflectances or to evaluate models. It is available freely from the PANGAEA website (doi:10.1594/PANGAEA.864090).

In addition to the database, we provide a visualization and analysis tool based on the Interactive Data Language (IDL). It allows an interactive analysis of the measurements and a comparison against various BRDF and BPDF analytical models. The present paper describes the input data, the selection principles, the database format, and the analysis tool.
\end{abstract}

\section{Introduction}

The albedo of a target is the fraction of the incoming light that is reflected rather than absorbed by the surface (Schaepman-Strub et al., 2006). It varies between zero (full absorption) and 1 (full reflection). The albedo of natural Earth targets varies widely depending on the surface types: vegetation absorbs most of the incoming visible light, whereas the opposite is true for snow. In addition, the albedo varies with wavelength. Many land surface characteristics can be inferred from the spectral signature of their albedo. Spectral indices such as the normalized difference vegetation index (NDVI) have been developed to quantify the amount and state of vegetation or other properties (Carlson and Ripley, 1997; Asrar et al., 1984).

The albedo is a quantity that integrates the reflected light over all directions of the hemisphere. This quantity is difficult to measure because a typical radiometer measures the reflected light in a single direction. This is particularly true for spaceborne observations where a target is observed from a given direction. As a direct consequence, the radiometer is not sensitive to the albedo but rather to the reflectance (Schaepman-Strub et al., 2006).

Global observation from space and multi-temporal monitoring of a given target impose the combination of mea- 
surements acquired with different observation geometries (Lunetta et al., 2006). It is well known that the reflectance of natural surfaces is far from isotropic (Bicheron and Leroy, 2000). For most land surfaces, the reflectance is larger in the backscatter direction than it is in the forward direction. In the very few degrees towards the backscatter direction, the reflectance increases markedly, an optical phenomenon referred to as the hot spot (Breon et al., 2002), but it is difficult to quantify properly from a surface-based apparatus because the measurement is perturbed by the radiometer's own shadow. Snow is much more isotropic than other surfaces, but nevertheless shows larger reflectance values in the forward rather than backward hemisphere, which is opposite to that of other land surfaces (Peltoniemi et al., 2005).

The directional signature of the reflectance is described by the bidirectional reflectance distribution function (BRDF) (Schaepman-Strub et al., 2006). In principle, it is a function of four angles, the illumination (solar) angle and view, zenith, and azimuth angles. In practice, and except for targets that show a preferential direction such as crops planted along rows, the two azimuths (solar and view) are only significant for the BRDF through their difference. Thus, the BRDF is most often described as a function of $\left(\theta_{\mathrm{s}}, \theta_{\mathrm{v}}, \phi\right)$, where $\theta_{\mathrm{s}}$ and $\theta_{\mathrm{v}}$ are the solar and view zenith angles respectively and $\varphi$ is the relative azimuth.

The goal of this paper is to describe a database of BRDF samples that has been developed based on spaceborne measurements of Earth reflectances. This database may be used to assess the variability of land surface BRDF for the development and validation of BRDF models and as a boundary condition for atmospheric radiative transfer studies.

Other characteristics of the land surface reflectance are its polarization properties. The incoming direct solar light is unpolarized. Conversely, the light scattered in the atmosphere by molecules and aerosols and the light reflected by the surface are partly polarized. Few optical instruments designed to monitor the Earth have polarization capabilities, and much less effort has been devoted to the polarization characterization of land surfaces than to the BRDF. However, polarization is a great tool to monitor anthropogenic aerosols and clouds from space, as demonstrated with the POLDER instrument (Waquet et al., 2009b; Deuze et al., 2001; Breon and Doutriaux-Boucher, 2005). This led to the development of the Glory mission (Mishchenko et al., 2007), which was unfortunately lost at launch. The 3MI instrument, which is similar to POLDER but with advanced capabilities in terms of spatial resolution and spectral coverage, shall be onboard the forthcoming series of Eumetsat MetOp satellites (Marbach et al., 2015). A primary objective of this space mission is the monitoring of atmospheric aerosols and clouds using the polarization characteristics of the reflected light.

Information about the land surface polarization characteristics is therefore needed. The database that is presented in this paper includes, in addition to the spectral reflectances, the polarization characteristics in one channel.
In the following, we describe the input data, the data processing and selection, and the database format. In addition, we have developed an interactive tool to allow a simple graphical analysis of the database and a comparison to analytical models. The tool is therefore described in the second part of the paper with a few examples of its outputs.

\section{Input data and processing}

\subsection{The POLDER instrument onboard the PARASOL mission}

The POLDER-1 and POLDER-2 radiometers were on-board the ADEOS 1 and 2 platforms in 1996-1997 and 2003 respectively (Deschamps et al., 1994). Unfortunately, the solar panel of both satellites failed after a few months of operations so that only 8 and 7 months of measurements were available from these instruments. This limitation did not allow the monitoring of a full vegetation cycle, which strongly reduced the interest in the data. Fortunately, a new opportunity occurred with the development by Centre national d'études spatiales (CNES) of a line of microsatellite platforms. The POLDER instrument was selected to be installed on-board one of these platforms and became a member of the ATrain to complement the other instruments. The satellite was named PARASOL after Polarization and Anisotropy of Reflectances for Atmospheric Sciences coupled with Observations from a Lidar (Tanré et al., 2011).

The experience gained with POLDER-1 and 2 was used and resulted in a few changes to the instrument, in particular regarding the choice of the spectral bands. There are eight spectral bands for the POLDER/PARASOL instrument, with central wavelengths from 443 to $1020 \mathrm{~nm}$ (Table 1). One main feature of POLDER is its capability for measuring the linear polarization of the light in three channels centred at 490,670 , and $865 \mathrm{~nm}$. This is achieved through three successive measurements with identical spectral filters and three polarizers rotated by steps of $60^{\circ}$. The processing of these measurements provides the radiance intensity, its polarization degree, and the polarization direction, or alternatively the Stokes vector components $(I, Q$, and $U)$.

The other main specificity of the POLDER instrument is its ability to provide multidirectional measurements. This is possible thanks to its optical design that consists of a wide field-of-view lens associated with a bi-dimensional CCD (charge-coupled device) matrix. This combination generates a bi-dimensional field of view with forward-backward angles of $\pm 51^{\circ}$ and cross-track angles of $\pm 43^{\circ}$. The maximum view angle at the surface is close to $70^{\circ}$ and corresponds to measurements acquired around the corners of the CCD matrix. As the satellite flies over, up to 16 (average is 14) observations of the target are available. These observations provide a sampling of the target BRDF. During the following days, the PARASOL satellite flies over that target again, albeit in a different orbit, which provides another set of BRDF sam- 
Table 1. POLDER/PARASOL spectral bands. The second line indicates the polarized channels, whereas the third line indicates the bands that are included in the BRDF-BPDF database.

\begin{tabular}{lrrrrrrrrr}
\hline Central wavelength & 443 & 490 & 565 & 670 & 763 & 765 & 865 & 910 & 1020 \\
\hline Polarization & $\mathrm{N}$ & $\mathrm{Y}$ & $\mathrm{N}$ & $\mathrm{Y}$ & $\mathrm{N}$ & $\mathrm{N}$ & $\mathrm{Y}$ & $\mathrm{N}$ & $\mathrm{N}$ \\
In database & $\mathrm{N}$ & $\mathrm{Y}$ & $\mathrm{Y}$ & $\mathrm{Y}$ & $\mathrm{N}$ & $\mathrm{Y}$ & $\mathrm{Y}$ & $\mathrm{N}$ & $\mathrm{Y}$ \\
\hline
\end{tabular}

ples. Depending on cloud cover, these successive measurements allow a very broad sampling of the BRDF for view angles up to $\approx 60^{\circ}$, assuming a stability of the target during the composition period. Note however that PARASOL is on a helio-synchronous orbit so that the various acquisitions are made at a near constant solar time. As a consequence, there is little variation in the sun angle in the measurements of the target reflectance during a short period.

The PARASOL satellite was launched in December 2004. Data acquisition started in early 2005 and was nearly continuous until October 2013. However, due to lack of fuel, the satellite left the A-Train and was on a slowly drifting orbit after December 2009. There have been some data acquisition interruptions during the lifetime of the satellite, mostly resulting from malfunctions of the stellar sensor. The best year in terms of data acquisition continuity was 2008. As a consequence, we selected that year to build the BRDF-BPDF (bidirectional polarization distribution function) database.

After the end of the in-orbit operations, POLDER/PARASOL data benefited from further development in calibration and data processing. Using several vicarious calibration techniques, all based on natural targets, it was possible to derive an accurate set of calibration parameters that account for the temporal evolution of the instrument sensitivity, characterized by a mean decrease modulated by a variation within the field of view (Fougnie, 2016). These developments led to a full reprocessing of the POLDER/PARASOL dataset at the end of 2015.

\subsection{POLDER data processing}

The POLDER instrument provides top-of-the-atmosphere reflectances after calibration (Fougnie et al., 2007). These Level-1 measurements are processed into Level-2 products using several processing chains. The reflectances are corrected for atmospheric absorption $\left(\mathrm{H}_{2} \mathrm{O}, \mathrm{O}_{3}, \mathrm{O}_{2}, \mathrm{NO}_{2}\right)$. Over land, the atmospheric aerosol load is estimated from the polarized reflectance measurements using precomputed tables (Deuze et al., 2001). The reflectance measurements are then corrected for atmospheric scattering for an estimate of the spectral surface reflectance. The polarized reflectances are corrected for the molecular scattering; they are not corrected for aerosol scattering.

The so-called Level-2-A official product contains an estimate of the directional surface reflectance for six spectral bands and an estimate of the directional surface polarized reflectance at $865 \mathrm{~nm}$. Only the longer wavelength channel is provided since it is generally assumed (and the POLDER aerosol inversion does so) that (i) the surface-polarized reflectance is spectrally neutral (Waquet et al., 2009a) and (ii) the atmospheric contribution is dominant and more difficult to correct for the shorter wavelength channels. The product also includes a nonquantitative indication of the aerosol load.

Some explanation is needed for what we refer to as the "polarized reflectance". As mentioned above, the POLDER instrument measures the Stokes vector representation $[I, Q$, $U]$ of the radiance. A reference plane is needed to define $Q$ and $U$. Many studies use the vertical plane (that contains the view and local nadir directions) as a reference. However, it is more practical to use the scattering plane (that contains the sun and view direction). With this plane as a reference, $U$ is most often very small with respect to both $I$ and $Q$ (Schutgens et al., 2004). This is because the polarization is either parallel or perpendicular to the plane of scattering. $Q$ is smaller than $I$ but takes measurable values. In most cases, the polarization is perpendicular to the plane of scattering so that $Q$ is negative. In rare cases, the polarization is parallel to the plane of scattering, in which case $Q$ is positive. We thus define the polarized reflectance $R_{\mathrm{p}}$ as

$R_{\mathrm{p}}=\frac{-\pi Q}{E_{0} \cos \theta_{\mathrm{s}}}$,

in a way similar to the reflectance definition

$R=\frac{\pi I}{E_{0} \cos \theta_{\mathrm{s}}}$,

where $E_{0}$ is the TOA (top of the atmosphere) solar irradiance. With such a definition, $R_{\mathrm{p}}$ is most often positive, but it nevertheless contains the information of whether polarization is perpendicular or parallel to the plane of scattering. In the following, the BRDF is the angular distribution of $R$, whereas the BPDF is the angular distribution of $R_{\mathrm{p}}$.

\subsection{Data selection}

The objective is to sample the variability of land surface BRDF and BPDF while selecting only the observations that are free from significant aerosol and cloud contamination and for which a large number of observations are available.

Early studies have shown that there are systematic changes in the BRDF with the land surface type (Bacour and Breon, 2005). As mentioned above, snow has a very specific directional signature; deserts show a more isotropic directional 
signature than vegetated surfaces, and wetlands sometimes show a glint signature in addition to the classical maximum in the backscatter direction. It is then natural to sample BRDFs as a function of the land surface cover. For this objective, we make use of the IGBP (International GeosphereBiosphere Programme) classification (Loveland et al., 1999). We used the official MODIS land cover product (MCD12Q1) for the year 2008 at $5 \mathrm{~min}$ resolution (Liang et al., 2015). For each POLDER pixel $\left(\approx 6.2 \times 6.2 \mathrm{~km}^{2}\right)$ we analyse the land cover type for the $5 \times 5$ MODIS cells centred on the POLDER pixel. Only the POLDER pixels for which there is a clear dominance of one land cover type $(>75 \%)$ are kept for further processing. The threshold was empirically chosen to ensure maximal homogeneity while keeping a representative spatial distribution of the selected targets. The POLDER pixels are assigned the IGBP land cover type as identified from the MODIS product, and the relative fraction of the dominant type is kept for inclusion as ancillary information in the database.

For each POLDER pixel that passes this first step, and for each of the 12 months independently, we retrieve all POLDER/PARASOL directional observations that pass the cloud detection scheme. A BRDF model is fitted against the $670 \mathrm{~nm}$ surface reflectances and the RMSE between measurements and the model is computed. The objective is to reject poorly corrected aerosol contamination, which increases the RMSE, and keep pixels with a large number of observations.

A score for the pixel month is defined as

$\operatorname{score}[p, m]=\sqrt{N_{\text {mes }}} / \mathrm{RMSE}$

where $p$ identifies the POLDER pixel and $m$ identifies the month; $N_{\text {mes }}$ is the number of directional POLDER measurements that are available. In addition, since there is a particular interest in the analysis of the hot spot directional signature, we increase the score by $20 \%$ if the set of directional measurements includes at least one with a phase angle of less than $1^{\circ}$. The angular threshold is the typical half width of the hot spot (Breon et al., 2002), whereas the score bonus value is an empirical choice based on the variability of the target scores.

We also compute a yearly score as the sum of the monthly scores:

$\operatorname{scoreY}[p]=\sum_{m} \operatorname{score}[p, m]$

For each IGBP surface type and each month, we select the 50 "best" targets, i.e. those that have the highest score. Conversely, we seek some diversity and thus want to avoid selecting pixels that are close to one another. We therefore select pixels iteratively: after a pixel with the highest score is selected, the score of all pixels is multiplied by $(1-\exp (d / 100))$, where $d$ is the distance (in $\mathrm{km}$ ) between each of the pixels and the pixel selected at the previous step.
The score of the nearby pixels is then reduced, which ensures that they are not subsequently selected.

As a result of this procedure, we independently select 50 targets for each of the 12 months and each of the 16 IGBP surface types. This procedure leads to the monthly database.

In addition, we generate a yearly database where the selection is based on the yearly score (scoreY) rather than the monthly scores. The procedure is very similar. In the yearly database, the same targets are selected for the 12 months. Conversely, the monthly database selects pixels independently for each month, which results in different target sets in most cases. The monthly database is best for analysing targets of high quality for each month independently. The yearly database shall be used to assess the variability of the BRDF and BPDF along the year, as shown in Sect. 3.7 below, although some months may be poorly sampled.

\subsection{Database structure}

The two databases (monthly and yearly) are built around a large number of text files $(\approx 16 \times 12 \times 50)$. Each file includes the surface reflectance and polarized reflectance acquired during the month. The files are sorted by IGBP surface types (nn from 01 to 16 ) and then by month ( $\mathrm{mm}$ from 01 to 12 ): The directory IGBP_nn contains the subdirectories $2008 \mathrm{~mm}$, which contain the files. The file format is described in Appendix A.

In addition, the database includes a binary file map_IGBP.bin. It reproduces the IGBP classification used for the data selection on a $540 \times 270$ (long $\times$ lat) grid. This file is used by the graphic analysis tool.

\section{Analyses of the database features using the visu_brdf analysis tool}

\subsection{Visu_brdf tool set-up}

A graphical interface tool has been developed to analyse the BRDF-BPDF data file described above. The code visu_brdf.pro is based on the IDL and its use requires an IDL licence. Another option, which does not require an IDL licence, is to download the IDL Virtual Machine from the Harris Geospatial website (https:/www.harris.com/ what-we-do/geospatial-solutions). The virtual machine lets you run the compiled version of the analysis tool, provided in the visu_brdf.sav file.

The first step is to locate the database. Inside the code, you change the variable "HomePath" to the directory that contains the monthly and yearly databases.

When using IDL with a proper licence, type

IDL > .compile visu_brdf.pro

IDL $>$ visu_brdf. 


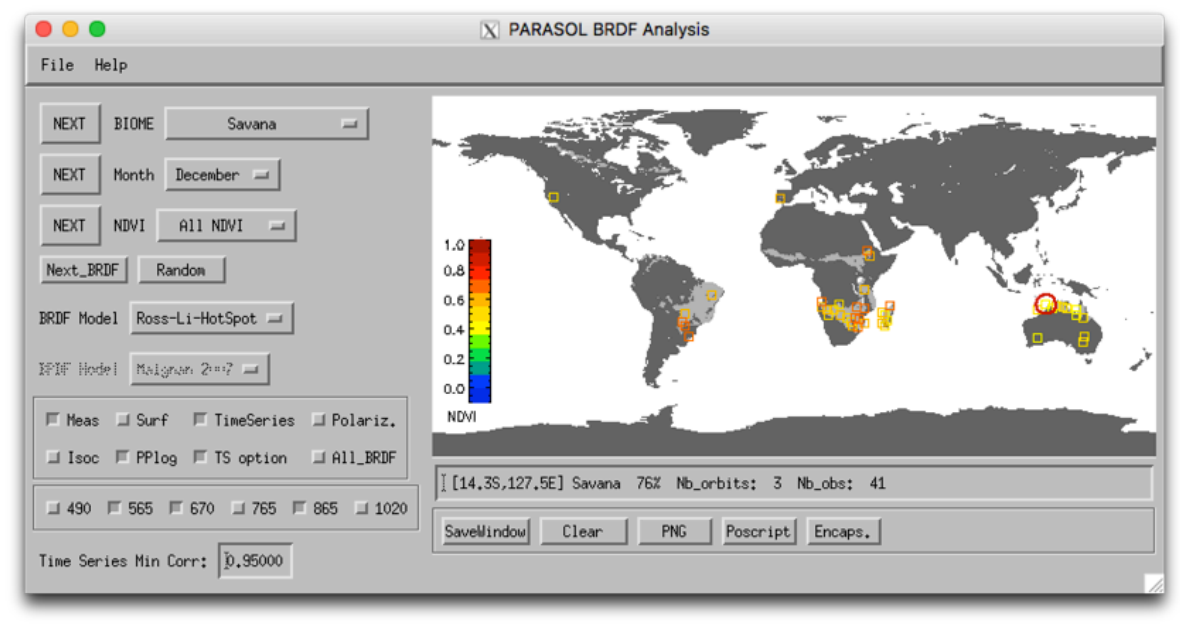

Figure 1. Main Command window (MCW) of the visu_BRDF analysis tool. Various buttons and drop-down menus permit the selection of a given target and various display options as described in the text. This version of the MCW is when the yearly database is used as input. When the monthly database is used, the MCW layout is slightly modified with fewer display options (i.e. TimeSeries, TS option, and All_BRDF are not shown).

If the HomePath was not set properly, a warning message indicates that one must select the path for the "POLDER BRDF" database and a window opens up for that purpose.

If one uses the IDL Virtual Machine option, just doubleclick on the visu_brdf.sav icon. As described above, one must then select the path for the database.

\subsection{The Main Command window}

Figure 1 shows the Main Command window of the BRDF analysis tool. In the following, it is referred to as MCW. One can select one of the IGBP surface types with the "BIOME" drop-down list or successive clicks on the "NEXT" button that is next to it. Similarly, one can select the time period with the "Month" drop-down list and the NDVI range with the "NDVI" drop-down list.

The available targets are shown on the map. Note the lighter grey areas that indicate the Earth surface that corresponds to the selected IGBP surface type. The squares indicate the locations of the targets in the BRDF database that correspond to the criteria (IGBP type, month, and NDVI range). According to the NDVI, the colour of the squares ranges from black to red (we use a rainbow palette) if "ALL NDVI" is selected or red if a specific range of NDVI is selected.

The selected target is indicated by a red circle. There are several ways to choose a target for display of its measurements. The easiest option is to click on the map close to the desired square. The other option is to use the "Next_BRDF" button that selects the various targets in successive order. Finally, it is also possible to use the "Random" button that selects a target randomly among the ones shown on the map.
Below the map is some information about the selected target: latitude and longitude, surface type, fraction of this surface type, the number of orbits (satellite overpasses), and the total number of observations for this target.

When a target is selected, several windows are displayed and can be changed with various options. They will be described below.

Below the BRDF and BPDF model selection is a box with check buttons that change the measurement model visualization. The Meas/Surf/Isoc checkboxes affect the Target BRDF window and are described below. The "Polariz" checkbox controls the display of a specific window for the BPDF. The "PPlog" checkbox toggles between a linear and log scale for the Principal Plane and Perpendicular Plane windows that are described below.

Further below, the checkboxes with the band central wavelengths make it possible to select three bands, out of the six provided in the database, for display in the Target BRDF, Principal Plane, and Perpendicular Plane windows.

Finally, the different buttons of the Main Command window located below the information about the target allow one to save a measurement-model comparison in various formats:

"PNG" saves the window in PNG format.

"Poscript" generates a poscript file.

"Encaps." generates encapsulated Poscript files (file.eps). Note that Figs. 2-7 have all been generated using this method. 


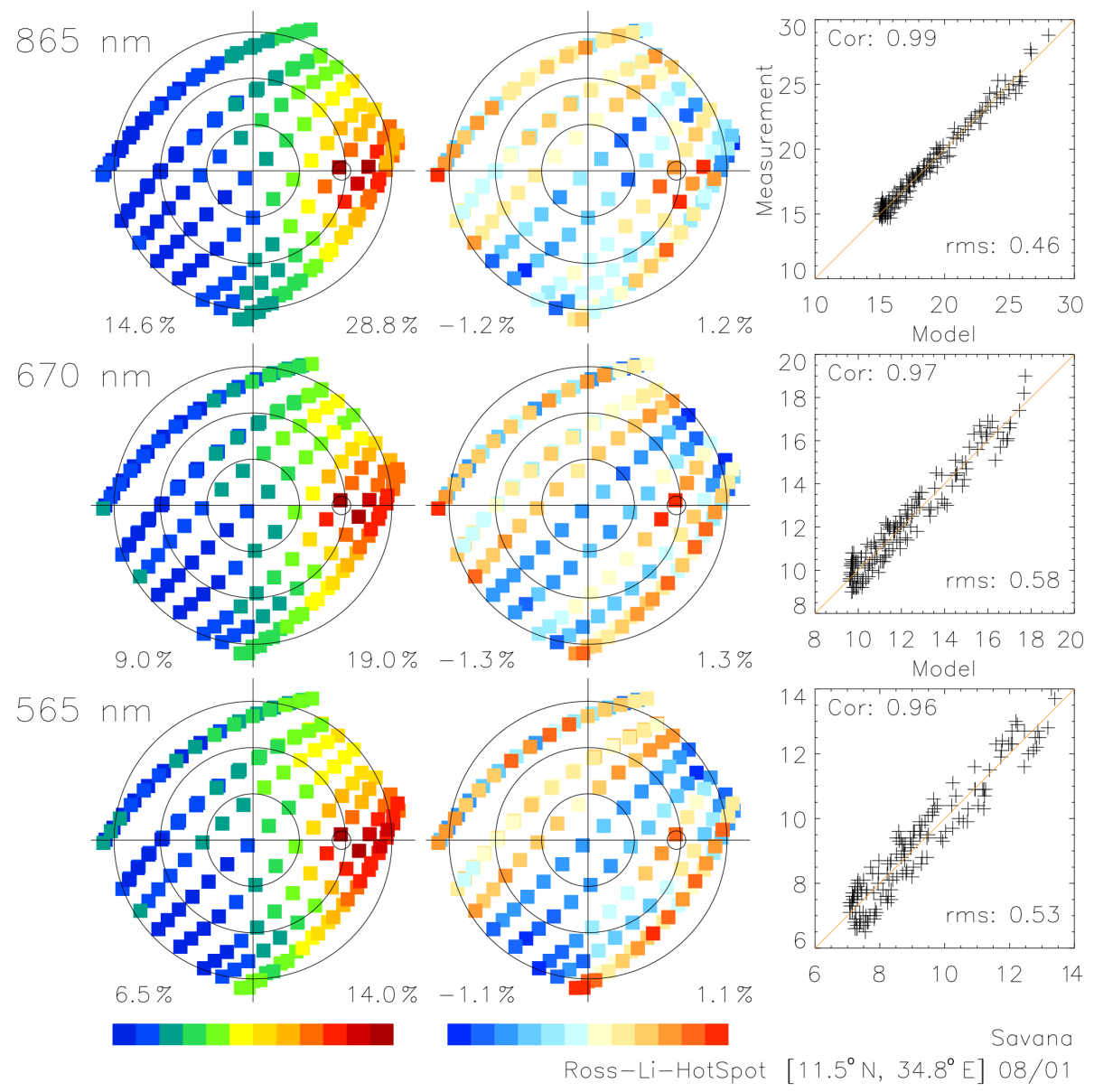

Figure 2. An example of the Target BRDF window content. The left column shows the measurements, the middle column shows the difference between the measurements and the modelling, and the left column is a measurement-model scatter plot. Three channels are shown, which can be selected out of six $(490,565,670,765,865$, and $1020 \mathrm{~nm})$. Note that the scales are different between channels.

The "SaveWindow" button makes a simple copy of the current window. This is useful for comparing the predictions of different models.

The "Clear" button erases all windows.

\subsection{BRDF-BPDF models within visu_brdf}

The visu_brdf tool can be used to compare the BRDF and/or BPDF measurements to analytical models. Several such models have been implemented within the tool and can be selected from the two drop-down lists "BRDF model" and "BPDF model". Currently, seven BRDF models are available in the visu_brdf tool:

Ross-Li (linear, three parameters): used in the MODIS processing (Schaaf et al., 2002),

Roujean (linear, three parameters): used in the original POLDER processing (Roujean et al., 1992),
Ross-Li HotSpot (linear, three parameters): with the hot spot modelling (Maignan et al., 2004),

Roujean HotSpot (linear, three parameters): with the hot spot modelling (Maignan et al., 2004),

Engelsen (semi-linear, three parameters) (Engelsen et al., 1998),

RPV (non-linear, three parameters) : model of RamanPinty-Verstraete (Verstraete et al., 1990),

Snow (linear, one parameter): specific for snow surfaces (Kokhanovsky and Breon, 2012).

Similarly, four BPDF models are available in the visu_brdf tool:

Nadal (linear, two parameters): used in early times of the POLDER experiment (Nadal and Breon, 1999),

Breon (linear, two parameters): two kernels developed for vegetation and bare soils (Breon et al., 1995), 

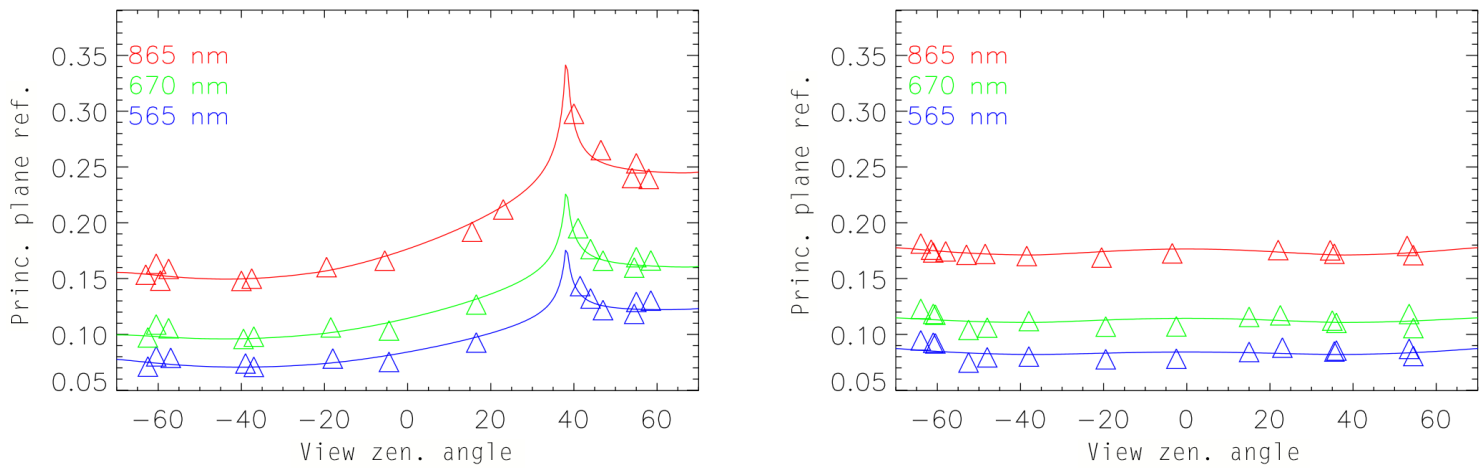

Figure 3. An example of the Principal Plane (left) and Perpendicular Plane (right) window contents. The measurements are shown as triangles and the model prediction is indicated as a line. Three channels are shown, which can be selected out of six. The $Y$ scale can be either linear or logarithmic depending on the MCW setting (Fig. 1).

Maignan (linear, one parameter): developed from POLDER measurements (Maignan et al., 2009),

Litvinov (non-linear, three parameters) (Litvinov et al., 2011).

There are plans to develop a BPDF model for snow surfaces, which may then become available for further releases of the visu_brdf tool.

\subsection{Analysis of a target BRDF}

Figure 2 shows an example of the Target BRDF window content. This window shows the reflectance measurements as well as a comparison against the modelling results after a best fit against the measurements. The BRDF model that is used is selected on the MCW. Each row of the figure corresponds to one chosen wavelength among the six available wavelengths $(490,565,670,765,865$, and $1020 \mathrm{~nm})$ in the bottom-left of the MCW.

The reflectances shown in Fig. 2 are typical for a surface with sparse vegetation. The reflectance is significantly larger in the near infrared $(865 \mathrm{~nm})$ than it is in the red $(670 \mathrm{~nm})$ or green $(565 \mathrm{~nm})$. For a given wavelength, the reflectance increases towards the backscatter direction. The model is able to reproduce most of the directional variation, as shown on the scatter plot (right column), and the model-measurement correlation is more than 0.97 . The central column shows the difference between measurement and modelled reflectances. In Fig. 2, these differences appear mostly random and do not show a systematic variation within the directional space. This indicates that there is little hope for a BRDF model that fits the measurement better. Other targets show measurementmodel differences with more spatial structure, indicating a deficiency in the modelling that might be improved (not shown).

By default, the left column shows the reflectance measurements as shown in Fig. 2. It is also possible to use other dis- plays of the measurements as selected with the toggle buttons in the MCW, which include the following options:

"Meas": shows the reflectance measurements (default),

"Isoc": shows the isolines of the model outputs (after a best fit),

"Surf": shows the modelled reflectance as a coloured surface. This option disables the two others.

The data are plotted on a polar diagram. The large circles correspond to view zenith angle of 20,40 , and $60^{\circ}$. The principal plane is on the horizontal line with backscatter to the right. The perpendicular plane is along the vertical line. The small circle on the horizontal line corresponds to the median sun angle during the period of synthesis and indicates the backscatter direction.

Note that the reflectance-difference ranges for the colour scales vary with the channel and are indicated at the bottom of each plot. Finally, information about the target is mentioned at the bottom of the figure: location, period, IGBP surface type, and analytical model used for comparison.

\subsection{Analysis of directional signatures in the principal and perpendicular planes}

Figure 3 presents an example of directional signatures in the principal and perpendicular planes. Such figures are automatically generated by the visu_brdf tool when a target and a model have been selected. The two windows show the measured (triangle symbols) and modelled (lines) reflectances in the principal and perpendicular planes for the three selected wavelengths as a function of the view zenith angle. Only those measurements close to the principal-perpendicular planes are displayed. The measurements are corrected for the BRDF variations between the geometry of observation and 

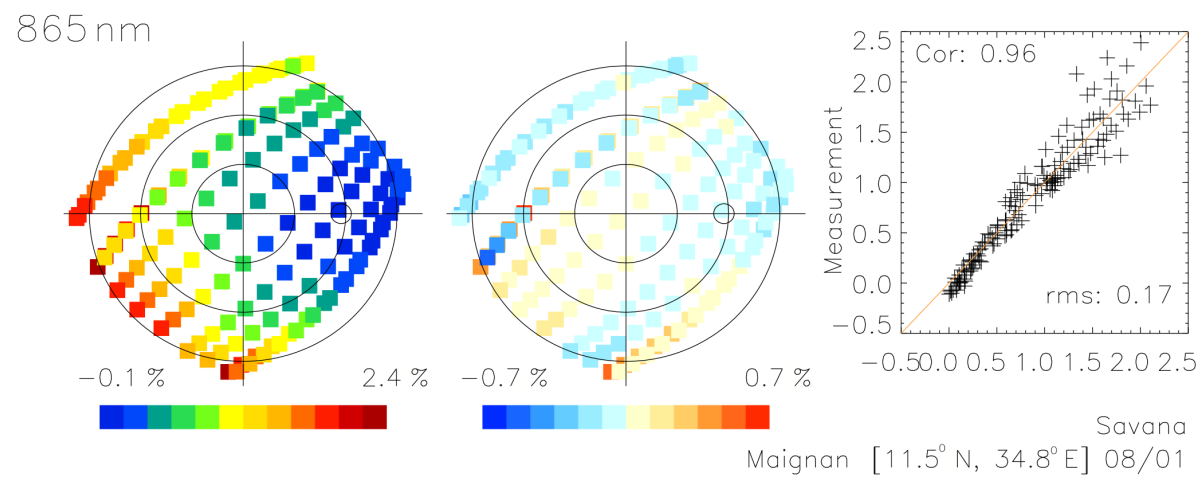

Figure 4. An example of the Target BPDF window contents. The presentation is similar to Fig. 2, but only the polarized reflectance at $865 \mathrm{~nm}$ is shown.

the principal-perpendicular plane, i.e. the symbols indicate

$$
\begin{gathered}
\text { Measurement }+ \text { Model (principal - perpendicular plane }) \\
- \text { Model(Viewing geometry) }
\end{gathered}
$$

This correction is small but it is necessary to account for the variation in the sun angle within the month and the small variations in the reflectance between the observation geometry and the parallel-perpendicular plane.

The $Y$ axis can be on either a linear or log scale depending on the "PPlog" option in the MCW. Similarly, the three channels that are displayed can be modified among the six that are available in the database. These figures confirm the general observation made from Fig. 2: the reflectance is significantly larger in the near-infrared than it is in the visible; it increases markedly from forward scatter towards backscatter, while there are insignificant variations in the perpendicular plane. The model accurately reproduces the observed variations.

\subsection{Analysis of a BPDF target}

Figure 4 shows an example of the content of the Target BPDF window, which is very similar to the Target BRDF except that it shows the surface polarized reflectance at a single wavelength $(865 \mathrm{~nm})$. Although the POLDER instrument made polarized measurements in three channels, only the longer wavelength channel is provided in the database and is therefore accessible through the visu_brdf tool. Our experience is that the surface polarized reflectance is spectrally neutral, or the spectral variations are smaller than the measurement noise. We thus provide the longer wavelength channel estimates that are the least contaminated by atmospheric scattering.

The left image shows the measurements, the middle image is the model-measurement difference, and the right figure is a scatter plot of the measurement and model. The same ancillary information as for the Target BRDF window is provided at the bottom of the window.
The directional signature of the polarized reflectance is completely different than for the reflectance. At backscatter, the polarized reflectance is very small and even negative, indicating a polarization parallel to the plane of scattering. The polarized reflectance tends to increase with the phase angle away from backscatter. Note that the polarized reflectance is much smaller than the reflectance, so that the polarization ratio is only a few percent. Although the model does a fairly good job, it does not reproduce the negative polarization close to backscatter. The scatter plot indicates two different regimes where the modelling is clearly larger or clearly smaller than the observation. The directional diagram (middle) does not show any systematic feature. Positive and negative differences are observed in very similar observation geometry. This indicates a slight change in the target polarized reflectance within the period of synthesis.

\subsection{Analysis of the temporal variations of the BRDF parameters using the yearly database}

As explained in the database description above, there are in fact two databases. The monthly database processes all months independently and selects the best targets for each month. Conversely, the yearly database selects a set of targets for the full year. With the latter, it is then possible to analyse how the BRDF and BPDF parameters vary during the year. To use the yearly database with the visu_brdf tool, either use the keyword /YEAR if the HomePath has been properly defined,

IDL > visu_brdf,/YEAR,

or provide the full path to the yearly database,

IDL $>$ visu_brdf, pathin $=$ '/home/users/breon/BRDF_database_ Year'. 
In such a case, the MCW is slightly different than the monthly database. There are three additional checkboxes below the drop-down buttons used for model selection.

These checkboxes, TimeSeries, TSoption, and All_BRDF, control the display of the windows that are described below. The TimeSeries checkbox must be selected to show the Vegetation Indices window and the Time Series window while TSoption controls its content. Similarly, the All_BRDF checkbox controls the display of the corresponding window.

\subsubsection{Vegetation Indices time series}

The Vegetation Indices window shows the time series of NDVI (in green) and $3 *$ DVI (in blue) over the full year. Rather than DVI, 3*DVI is shown to get a range similar to that of NDVI. DVI is the simple difference of the 865 and $670 \mathrm{~nm}$ channel reflectances $\left(\mathrm{DVI}=\left(R_{865}-R_{670}\right)\right)$. NDVI is the normalized difference of these parameters (NDVI $=$ $\left.\left(R_{865}-R_{670}\right) /\left(R_{865}+R_{670}\right)\right)$. The reflectance is the nadir value derived from the selected BRDF model after a fit to the measurements. The purpose of the Vegetation Indices window is mostly to provide some indication about the vegetation cover variations within the year for a better interpretation of the figures that are discussed below. On the example shown in Fig. 5, there is a clear annual cycle of the vegetation cover with an increase in the vegetation indices during the spring and a dry down during the fall.

\subsubsection{Model parameter time series}

The Time Series window displays the annual time series of the three parameters of a linear BRDF model, referred to as $k_{0}, k_{1}$, and $k_{2}$. The model used is that selected in the MCW but, in the current version the Time Series window only functions for the linear BRDF models (i.e. not Engelsen, RPV, and Snow). If the TS Option checkbox is set, it displays the time series of the reflectance in a particular geometry (sun at $40^{\circ}$ from zenith and view at nadir) from top to bottom and the ratio of model parameters $k_{1} / k_{0}$, and $k_{2} / k_{0}$. An example is shown on the right side in Fig. 6. When the checkbox is not set, the time series of $k_{0}, k_{1}$, and $k_{2}$ are shown, as on the left side in Fig. 6. The time series of the three selected channels are shown in colour, while the others channels are also displayed but in black. Note that the parameters are displayed only if the coefficient of correlation between measurements and the model is larger than the "Time Series Min. Corr" threshold set in the MCW. Indeed, when the correlation is low, the BRDF coefficients have little value and should not be displayed. The user can change the threshold and see its influence on the results. Note that the ratios of the model parameters quantify the overall shape of the BRDF directional variation. It has been shown that the temporal variations of a target reflectance are mostly driven by the BRDF amplitude rather than its shape, whose variations are slower (Breon and Vermote, 2012). In the single-scattering approximation

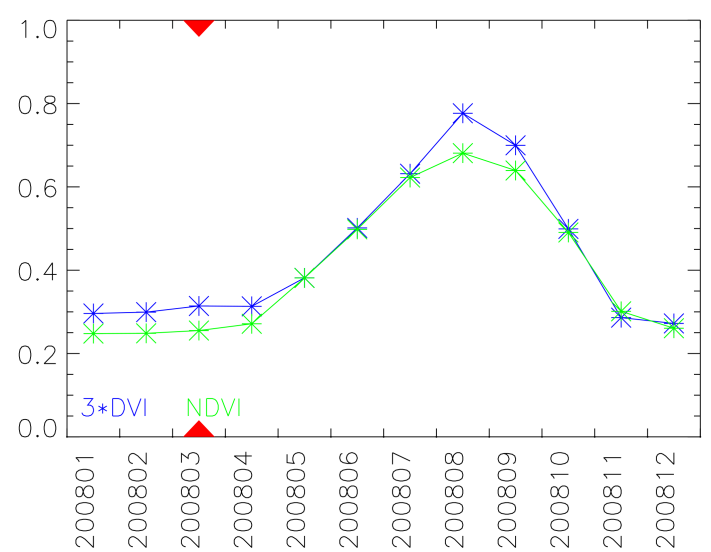

Figure 5. An example of the NDVI window content. It shows the time series of the NDVI and the DVI. The red triangles indicate the month that is displayed in other windows (Figs. 2-4).

(which is almost valid in the visible since the reflectance is rather low), the directional effects are generated by the target architecture, while its overall amplitude depends on the reflectance of the individual elements. As a consequence, the BRDF can be expressed as the product of a normalized reflectance and a normalized shape. The visu_brdf tool thus provides the option to display the parameter ratios in addition to their plain values.

The example of the Time Series window shown in Fig. 6 indicates that the target BRDF changes with the vegetation growth and decay. Indeed, $k_{1}$ and $k_{2}$ (left), or their ratio with $k_{0}$ (right), vary concomitantly with the vegetation indices. Although it is not seen for all bands, $k_{1}$ tends to decrease with an increase in the vegetation index, while $k_{2}$ tends to increase. This behaviour is found over most targets (Breon and Vermote, 2012) and is somewhat expected since $k_{2}$ is associated with the RossThick kernel, which aims at modelling the BRDF of a thick canopy. Note that the time series are often incomplete because of a lack of observations (because of cloud cover or sun angle issues). In the example in Fig. 6, there are no parameter estimates for December.

The time series in this example are relatively clean. There are cases with more variable parameter retrievals. The blue $(490 \mathrm{~nm})$ band over the vegetation is particularly difficult because of the low surface signal and the large atmospheric correction.

\subsubsection{BRDF-BPDF seasonal evolution}

Finally, when the All_BRDF checkbox is set, the All BRDF$B P D F$ window shows the BPDF (first line) and BRDF (following lines) for the 12 months. An example is shown in Fig. 7. In each of the polar diagrams that are shown, one can identify the independent satellite overpasses, with up to 16 observation directions that are roughly aligned in the angular space. The general orientation of these observations varies 

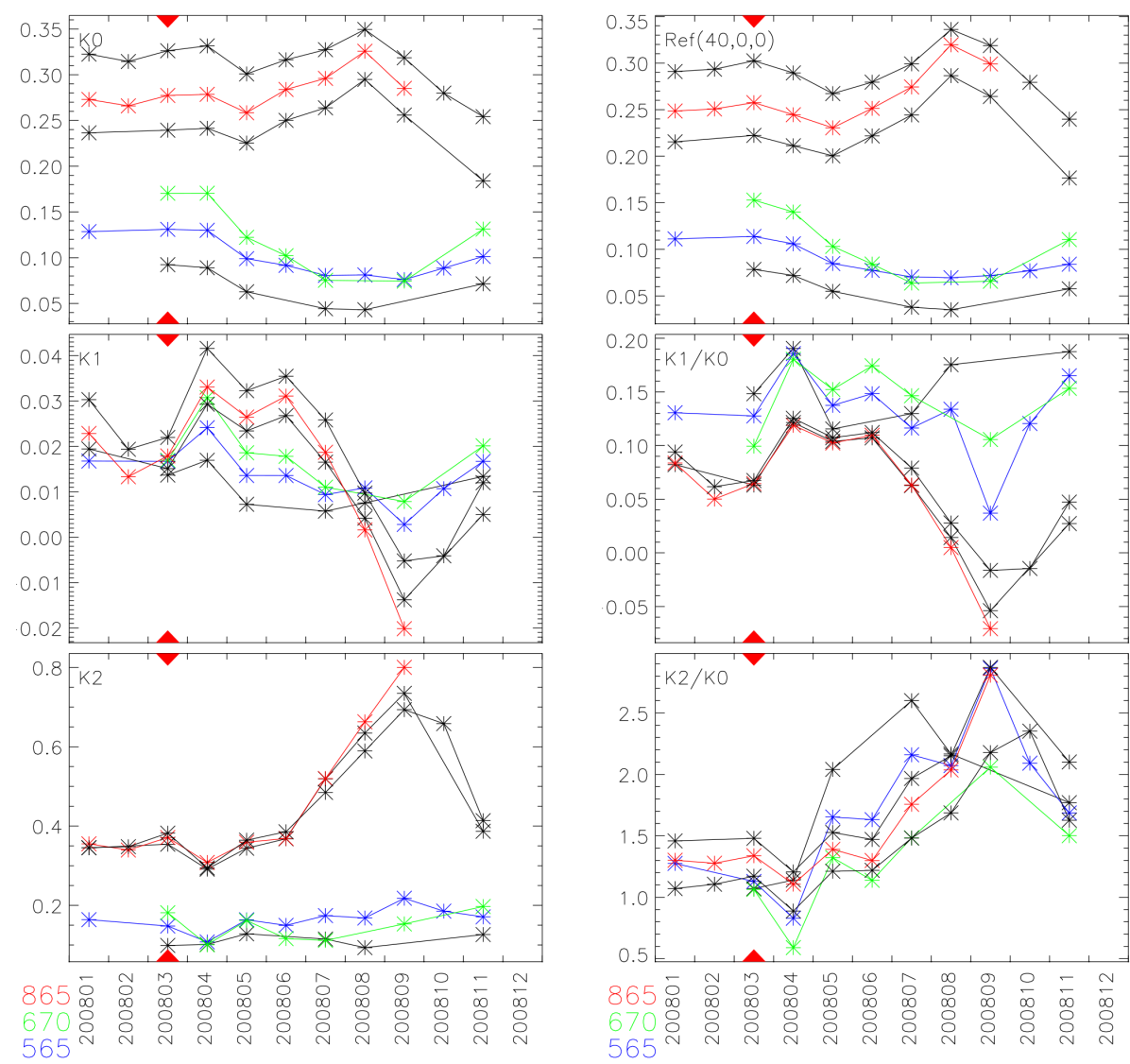

Figure 6. Example of the Time Series window content. Depending on the setting of the MCW, this window shows either the monthly time series of the BRDF model parameters (left) or a combination of the same. For the latter option, the top figure is the reconstructed reflectance for a reference observation geometry. In these figures, the coloured line or symbols are for the three selected bands, while the same parameters for the other bands are also shown in black.

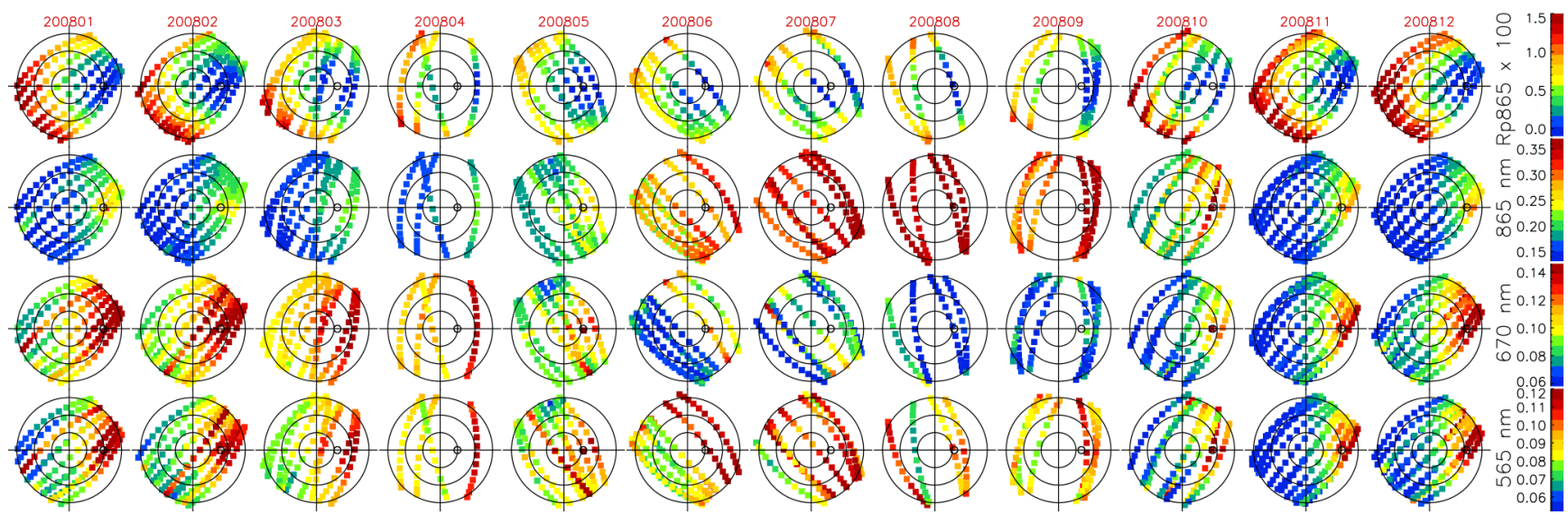

Figure 7. An example of the content of the All BRDF-BPDF window. This window shows the measurements for all 12 months in the database for a given target. The top line is the BPDF at $865 \mathrm{~nm}$, while the other three lines are for the reflectances for the three selected bands out of six. 
during the year because the sun azimuth varies at the local time observation.

All polar plots show the main characteristics that were described earlier, with a maximum reflectance and a minimum polarized reflectance close to backscatter. Figure 8 also shows a change in the general reflectance during the year. At $865 \mathrm{~nm}$ the reflectance is the largest in August, when it appears to be the lowest at $670 \mathrm{~nm}$. This observation is fully consistent with the change in the vegetation index (Fig. 5) and the BRDF parameters (Fig. 6) during the year. The All BRDF-BPDF window is appropriate to get a full view of the observation of a given target during the year, while the other windows are required for a more quantitative interpretation.

\section{Conclusions}

The main focus of the POLDER spaceborne instrument was for atmospheric studies, i.e. the monitoring of aerosols and clouds. It may be argued that the spatial resolution of the instrument, $6 \times 6 \mathrm{~km}^{2}$, is not suitable for the analysis of land surface processes. Indeed, most landscapes show significant variability within a target of such a surface. The averaging may then smooth out some specific BRDF features. We have attempted here to select homogeneous targets, in which case the spatial resolution of the measurement is not an issue. The analysis of BRDF derived from airborne measurements on a finer scale (Gatebe and King, 2016) does not reveal specific features that are not present in the database described here. We thus argue that our database does describe the variability of land surface BRDF since there is presently no evidence of a significant scale effect. A full comparison of the BRDF database described in Gatebe and King (2016) and that described here may be necessary to quantify the potential scale effect on the BRDF.

Although the first version of the instrument was launched 20 years ago (1996 on the ADEOS-1 platform), it remains the only instrument that measures the full linear polarization of the Earth reflectance in the solar domain. Addition- ally, the directionalcoverage of POLDER is better than that of multiangle imaging spectroradiometer (MISR) (Diner et al., 1998), the only other instrument that provides multidirectional sampling of the Earth reflectances (Lallart et al., 2008). Therefore, POLDER remains an up-to-date tool for the analysis of the directionality and polarization of land surface reflectances. We developed a database for the remote sensing community that provides a description of representative Earth targets. A similar undertaking was achieved based on airborne measurements at a higher spatial resolution (Gatebe and King, 2016). Earlier version of the database were developed based on the measurements from the POLDER instrument onboard the ADEOS and PARASOL satellites. Although these versions have not been properly described in the peer-reviewed literature, they have been used for several analyses of the surface directional (e.g. Kokhanovsky and Breon, 2012; Cui et al., 2009; Jiao et al., 2014; Bacour and Breon, 2005; Maignan et al., 2004) and polarization (e.g. Litvinov et al., 2012; Maignan et al., 2009) signatures. The new version, which is described in this paper, is of better quality. It benefits from improved calibration and data selection scheme, provides the reflectance measurement over an extended spectral range (up to $1020 \mathrm{~nm}$ ), and is associated with an interactive analysis tool. These data can be used to develop new models and evaluate their ability to reproduce the observed spectral, directional, and polarization signatures.

\section{Data availability}

The database and the analysis tool are available free of charge for the scientific community from the PANGAEA website (doi:10.1594/PANGAEA.864090). The POLDER/PARASOL Level-1 and Level-2 products were generated by CNES and are distributed by ICARE (http://icare.univ-lille1.fr). 


\begin{tabular}{|c|c|c|c|c|c|c|c|c|c|c|c|c|c|c|c|}
\hline latituo & long & gitude & IGB & BP_clas & & JDVI & orbit & nb_dir & homoge & eneity & $\left(\frac{\circ}{0}\right)$ & & & & \\
\hline 65.4 & & 119.58 & & 3 & & .32 & 15 & 210 & 100 & & & & & & \\
\hline yymmdd & Orbit & $\mathrm{SZA}$ & VZA & RelAzi & Azis & DVzC & DVzS & R490 & R565 & R670 & R765 & R865 & $R 1020$ & Rp865 & Aero \\
\hline 080307 & 075061 & 70.7 & 59.2 & 15.8 & 186.0 & -0.068 & -0.041 & 0.394 & 0.374 & 0.372 & 0.400 & 0.386 & 0.378 & 0.0012 & 2 \\
\hline 080307 & 075061 & 70.7 & 54.3 & 14.4 & 186.0 & -0.074 & -0.044 & 0.390 & 0.367 & 0.360 & 0.387 & 0.381 & 0.358 & 0.0005 & 2 \\
\hline 080307 & 075061 & 70.7 & 48.6 & 12.4 & 186.0 & -0.088 & -0.052 & 0.376 & 0.378 & 0.364 & 0.360 & 0.367 & 0.339 & 0.0031 & 2 \\
\hline 080307 & 075061 & 70.7 & 41.9 & 9.4 & 186.0 & -0.106 & -0.060 & 0.360 & 0.348 & 0.341 & 0.353 & 0.361 & 0.325 & 0.0067 & 2 \\
\hline 080307 & 075061 & 70.7 & 34.0 & 4.6 & 186.0 & -0.127 & -0.069 & 0.347 & 0.342 & 0.341 & 0.352 & 0.352 & 0.316 & 0.0065 & 2 \\
\hline
\end{tabular}

Figure A1. The first lines of a BRDF-BPDF data file.

\section{Appendix A: File structure}

Each file contains all PARASOL measurements for a target acquired during a month. The name of the file "brdf_ndviNN_LLLL_CCCC.txt" indicates its NDVI range and its location:

NN indicates the NDVI range.

LLLL is the line number in the POLDER sinusoidal grid (1 to 3240 ).

CCCC is the column number in the POLDER sinusoidal grid (1 to 6480).

The first lines of a BRDF-BPDF data file are given in Fig. A1.

Each file starts with three header lines. The header provides the pixel location in latitude and longitude, the IGBP class number, the NDVI, the number of valid satellite overpasses, the number of valid observations (each overpass provides up to 16 different directional measurements), and the fraction of the dominant surface type within the POLDER pixel (about $6 \times 6 \mathrm{~km}^{2}$ ).

The format of the second line of the header that contains the numerical values is

$$
\begin{aligned}
\mathrm{fmtHead}= & (F 8.2,3 x, F 8.2,5 x, I 3,4 x, F 7.2,5 x, I 3,5 x, \\
& I 4,6 x, I 3)^{\prime} .
\end{aligned}
$$

The header is followed by the measurements. Each line corresponds to one directional observation. The fill value, indicating an absence of observation, is -9.990 . The format of the different columns is

$$
\begin{aligned}
\text { fmtLine }= & (I 6, x, I 6,2 F 5.1,2 F 6.1,2 F 7.3,2 x, \\
& 6 F 6.3, F 8.4, I 3)^{\prime} .
\end{aligned}
$$

Each line contains the following information:

yymmdd is the date of observation.

Orbit provides the PARASOL orbit "cccooo" where "ccc" is the PARASOL cycle number $(1<\mathrm{ccc}<999)$ and "ooo" is the orbit number $(1<$ ooo $<233)$.

SZA is the sun zenith angle in degrees.

VZA is the view zenith angle in degrees.

RelAzi is the relative azimuth in degrees.

AziS is the sun azimuth with respect to north, in degrees.

DVzC and DVzS can be used for slight corrections of the view geometry. Indeed, POLDER spectral measurements are not simultaneous, meaning each channel is acquired with a slightly different viewing geometry. The view angles that are given are for the $670 \mathrm{~nm}$ band. The view geometry for the other channels can vary by a few tenths of degrees. For applications that require a higher accuracy, these parameters allow the correction that is described in Appendix B.

RXXX are the surface reflectances at 490, 565, 670, 765, 865 , and $1020 \mathrm{~nm}$.

Rp865 is the surface-polarized reflectance at $865 \mathrm{~nm}$. Positive values indicate a polarization perpendicular to the plane of scattering. Negative values indicate a polarization parallel to the plane of scattering. A further explanation can be found in Sect. 2.2.

Aero is a nonquantitative indication of the aerosol load retrieved from POLDER measurements. Zero is for minimal aerosol load, whereas 15 is for a high aerosol load. 
Table B1.

\begin{tabular}{lrrrrrrrrr}
\hline$X_{j}=$ & -6 & -4 & -3 & -2 & 0 & 2 & 3 & 4 & 6 \\
\hline Channel & $490 \mathrm{P}$ & 443 & 1020 & 565 & 670 & 763 & 765 & 910 & 865 \\
\hline
\end{tabular}

Appendix B: Compute the exact view direction for all channels

With the POLDER/PARASOL imaging concept, the 15 spectral-polarized measurements are acquired sequentially. Therefore, a given surface target is observed for the various spectral bands with slightly different viewing angles. The differences are small but can be significant for some applications that need a very high angular accuracy, such as the analysis of the hot spot directional signature.

The view zenith angle $\left(\theta_{0}\right)$ and relative azimuth $\left(\varphi_{0}\right)$ that are given in the BRDF database are for the central filter, i.e. 670P2. The two parameters $\mathrm{DVzC}=\Delta\left[\theta_{\mathrm{v}} \cos (\varphi)\right]$ and $\mathrm{DVzS}=\Delta\left[\theta_{\mathrm{v}} \sin (\varphi)\right]$, which are given for each viewing direction in the data file, are necessary for deriving these angles for other spectral bands $\theta_{j}$ and $\phi_{j}$. The formulae are as follows:

$\theta_{j}={\sqrt{\left(\theta_{0} \cos \phi_{0}+X_{j} \mathrm{DVzC}\right)^{2}+\left(\theta_{0} \sin \phi_{0}+X_{j} \mathrm{DVzS}\right)}}^{2}$

$\phi_{j}=\arctan \left(\frac{\theta_{0} \sin \phi_{0}+X_{j} \mathrm{DVzS}}{\theta_{0} \cos \phi_{0}+X_{j} \mathrm{DVzC}}\right)$.

If $\theta_{0} \sin \phi_{0}+X_{j} \mathrm{DVzS}<0$ then $\phi_{j}=\phi_{j}+180^{\circ}$, where $X_{j}$ is given in Table B1.

Note that this formulation is based on the simple principle that the 15 measurements are acquired with roughly equal spacing and on a straight line in an angular system of orthogonal axes $(\theta \cos \phi, \theta \sin \phi)$. 
Competing interests. The authors declare that they have no conflict of interest.

Acknowledgement. The work that led to this database was made possible thanks to the support from CNES and Eumetsat.

Edited by: D. Carlson

Reviewed by: two anonymous referees

\section{References}

Asrar, G., Fuchs, M., Kanemasu, E. T., and Hatfield, J. L.: Estimating Absorbed Photosynthetic Radiation and Leaf-Area Index from Spectral Reflectance in Wheat, Agron. J., 76, 300-306, 1984.

Bacour, C. and Breon, F. M.: Variability of biome reflectance directional signatures as seen by POLDER, Remote Sens. Environ., 98, 80-95, doi:10.1016/j.rse.2005.06.008, 2005.

Bicheron, P. and Leroy, M.: Bidirectional reflectance distribution function signatures of major biomes observed from space, J. Geophys. Res.-Atmos., 105, 26669-26681, doi:10.1029/2000jd900380, 2000.

Breon, F. M. and Doutriaux-Boucher, M.: A comparison of cloud droplet radii measured from space, IEEE T. Geosci. Remote, 43, 1796-1805, doi:10.1109/Tgrs.2005.852838, 2005.

Breon, F. M. and Vermote, E.: Correction of MODIS surface reflectance time series for BRDF effects, Remote Sens. Environ., 125, 1-9, doi:10.1016/J.Rse.2012.06.025, 2012.

Breon, F. M., Tanre, D., Lecomte, P., and Herman, M.: Polarized Reflectance of Bare Soils and Vegetation - Measurements and Models, IEEE T. Geosci. Remote, 33, 487-499, doi:10.1109/36.377949, 1995.

Breon, F. M., Maignan, F., Leroy, M., and Grant, I.: Analysis of hot spot directional signatures measured from space, J. Geophys. Res.-Atmos., 107, 4282, doi:10.1029/2001jd001094, 2002.

Carlson, T. N. and Ripley, D. A.: On the relation between NDVI, fractional vegetation cover, and leaf area index, Remote Sens. Environ., 62, 241-252, doi:10.1016/S0034-4257(97)00104-1, 1997.

Cui, Y., Mitomi, Y., and Takamura, T.: An empirical anisotropy correction model for estimating land surface albedo for radiation budget studies, Remote Sens. Environ., 113, 24-39, doi:10.1016/j.rse.2008.08.007, 2009.

Deschamps, P. Y., Breon, F. M., Leroy, M., Podaire, A., Bricaud, A., Buriez, J. C., and Seze, G.: The Polder Mission - Instrument Characteristics and Scientific Objectives, IEEE T. Geosci. Remote, 32, 598-615, doi:10.1109/36.297978, 1994.

Deuze, J. L., Breon, F. M., Devaux, C., Goloub, P., Herman, M., Lafrance, B., Maignan, F., Marchand, A., Nadal, F., Perry, G., and Tanre, D.: Remote sensing of aerosols over land surfaces from POLDER-ADEOS-1 polarized measurements, J. Geophys. Res.-Atmos., 106, 4913-4926, doi:10.1029/2000jd900364, 2001.

Diner, D. J., Beckert, J. C., Reilly, T. H., Bruegge, C. J., Conel, J. E., Kahn, R. A., Martonchik, J. V., Ackerman, T. P., Davies, R., Gerstl, S. A. W., Gordon, H. R., Muller, J. P., Myneni, R. B.,
Sellers, P. J., Pinty, B., and Verstraete, M. M.: Multi-angle Imaging SpectroRadiometer (MISR) - Instrument description and experiment overview, IEEE T. Geosci. Remote, 36, 1072-1087, doi:10.1109/36.700992, 1998.

Engelsen, O., Pinty, B., Verstraete, M. M., and Martonchik, J. V.: Parametric surface bidirectional reflectance factor models for atmospheric radiative transfer modeling, Igarss'98 - 1998 International Geoscience and Remote Sensing Symposium, Volume 1-5, 713-715, doi:10.1109/Igarss.1998.699559, 1998.

Fougnie, B.: Improvement of the PARASOL Radiometric In-Flight Calibration Based on Synergy Between Various Methods Using Natural Targets, IEEE T. Geosci. Remote, 54, 2140-2152, doi:10.1109/Tgrs.2015.2496322, 2016.

Fougnie, B., Bracco, G., Lafrance, B., Ruffel, C., Hagolle, O., and Tinell, C.: PARASOL in-flight calibration and performance, Appl. Optics, 46, 5435-5451, doi:10.1364/Ao.46.005435, 2007.

Gatebe, C. K. and King, M. D.: Airborne spectral BRDF of various surface types (ocean, vegetation, snow, desert, wetlands, cloud decks, smoke layers) for remote sensing applications, Remote Sens. Environ., 179, 131-148, doi:10.1016/j.rse.2016.03.029, 2016.

Jiao, Z. T., Dong, Y. D., Zhang, H., and Li, X. W.: To Derive Brdf Archetypes from Polder-3 Brdf Database, Int. Geosci. Remote Se., 3586-3589, doi:10.1109/Igarss.2014.6947258, 2014.

Kokhanovsky, A. A. and Breon, F. M.: Validation of an Analytical Snow BRDF Model Using PARASOL Multi-Angular and Multispectral Observations, IEEE Geosci. Remote S., 9, 928-932, doi:10.1109/Lgrs.2012.2185775, 2012.

Lallart, P., Kahn, R., and Tanre, D.: POLDER2/ADEOSII, MISR, and MODIS/Terra reflectance comparisons, J. Geophys. Res.Atmos., 113, D14s02, doi:10.1029/2007jd009656, 2008.

Liang, D., Zuo, Y., Huang, L. S., Zhao, J. L., Teng, L., and Yang, F.: Evaluation of the Consistency of MODIS Land Cover Product (MCD12Q1) Based on Chinese $30 \mathrm{~m}$ GlobeLand30 Datasets: A Case Study in Anhui Province, China, ISPRS Int. J. Geo-Inf., 4, 2519-2541, doi:10.3390/ijgi4042519, 2015.

Litvinov, P., Hasekamp, O., and Cairns, B.: Models for surface reflection of radiance and polarized radiance: Comparison with airborne multi-angle photopolarimetric measurements and implications for modeling top-of-atmosphere measurements, Remote Sens. Environ., 115, 781-792, doi:10.1016/j.rse.2010.11.005, 2011.

Litvinov, P., Hasekamp, O., Dubovik, O., and Cairns, B.: Model for land surface reflectance treatment: Physical derivation, application for bare soil and evaluation on airborne and satellite measurements, J. Quant. Spectrosc. Ra., 113, 2023-2039, doi:10.1016/j.jqsrt.2012.06.027, 2012.

Loveland, T. R., Zhu, Z. L., Ohlen, D. O., Brown, J. F., Reed, B. C., and Yang, L. M.: An analysis of the IGBP global land-cover characterization process, Photogramm. Eng. Rem. S., 65, 10211032, 1999.

Lunetta, R. S., Knight, J. F., Ediriwickrema, J., Lyon, J. G., and Worthy, L. D.: Land-cover change detection using multitemporal MODIS NDVI data, Remote Sens. Environ., 105, 142154, doi:10.1016/j.rse.2006.06.018, 2006.

Maignan, F., Breon, F. M., and Lacaze, R.: Bidirectional reflectance of Earth targets: Evaluation of analytical models using a large set of spaceborne measurements with empha- 
sis on the Hot Spot, Remote Sens. Environ., 90, 210-220, doi:10.1016/j.rse.2003.12.006, 2004.

Maignan, F., Breon, F. M., Fedele, E., and Bouvier, M.: Polarized reflectances of natural surfaces: Spaceborne measurements and analytical modeling, Remote Sens. Environ., 113, 2642-2650, doi:10.1016/j.rse.2009.07.022, 2009.

Marbach, T., Riedi, J., Lacan, A., and Schlussel, P.: The 3MI Mission: Multi-Viewing -Channel -Polarisation Imager of the EUMETSAT Polar System: Second Generation (EPS-SG) dedicated to aerosol and cloud monitoring, P. Soc. Photo-Opt. Ins., 9613, 961310, doi:10.1117/12.2186978, 2015.

Mishchenko, M. I., Cairns, B., Kopp, G., Schueler, C. F., Fafaul, B. A., Hansen, J. E., Hooker, R. J., Itchkawich, T., Maring, H. B., and Travis, L. D.: Accurate monitoring of terrestrial aerosols and total solar irradiance - Introducing the glory mission, B. Am. Meteorol. Soc., 88, 677-691, doi:10.1175/BAMS-88-5-677, 2007.

Nadal, F. and Breon, F. M.: Parameterization of surface polarized reflectance derived from POLDER spaceborne measurements, IEEE T. Geosci. Remote, 37, 1709-1718, doi:10.1109/36.763292, 1999.

Peltoniemi, J. I., Kaasalainen, S., Naranen, J., Matikainen, L., and Piironen, J.: Measurement of directional and spectral signatures of light reflectance by snow, IEEE T. Geosci. Remote, 43, 22942304, doi:10.1109/Tgrs.2005.855131, 2005.

Roujean, J. L., Leroy, M., and Deschamps, P. Y.: A Bidirectional Reflectance Model of the Earths Surface for the Correction of Remote-Sensing Data, J. Geophys. Res.-Atmos., 97, 2045520468, 1992.

Schaaf, C. B., Gao, F., Strahler, A. H., Lucht, W., Li, X. W., Tsang, T., Strugnell, N. C., Zhang, X. Y., Jin, Y. F., Muller, J. P., Lewis, P., Barnsley, M., Hobson, P., Disney, M., Roberts, G., Dunderdale, M., Doll, C., d'Entremont, R. P., Hu, B. X., Liang, S. L., Privette, J. L., and Roy, D.: First operational BRDF, albedo nadir reflectance products from MODIS, Remote Sens. Environ., 83, 135-148, doi:10.1016/S0034-4257(02)00091-3, 2002.
Schaepman-Strub, G., Schaepman, M. E., Painter, T. H., Dangel, S., and Martonchik, J. V.: Reflectance quantities in optical remote sensing-definitions and case studies, Remote Sens. Environ., 103, 27-42, doi:10.1016/j.rse.2006.03.002, 2006.

Schutgens, N. A. J., Tilstra, L. G., Stammes, P., and Breon, F. M.: On the relationship between Stokes parameters $Q$ and $U$ of atmospheric ultraviolet/visible/near-infrared radiation, J. Geophys. Res.-Atmos., 109, D09205, doi:10.1029/2003jd004081, 2004.

Tanré, D., Bréon, F. M., Deuzé, J. L., Dubovik, O., Ducos, F., François, P., Goloub, P., Herman, M., Lifermann, A., and Waquet, F.: Remote sensing of aerosols by using polarized, directional and spectral measurements within the A-Train: the PARASOL mission, Atmos. Meas. Tech., 4, 1383-1395, doi:10.5194/amt-4-1383-2011, 2011.

Verstraete, M. M., Pinty, B., and Dickinson, R. E.: A Physical Model of the Bidirectional Reflectance of Vegetation Canopies - 1. Theory, J. Geophys. Res.-Atmos., 95, 11755-11765, doi:10.1029/JD095iD08p11755, 1990.

Waquet, F., Leon, J. F., Cairns, B., Goloub, P., Deuze, J. L., and Auriol, F.: Analysis of the spectral and angular response of the vegetated surface polarization for the purpose of aerosol remote sensing over land, Appl. Opt., 48, 1228-1236, doi:10.1364/Ao.48.001228, 2009a.

Waquet, F., Riedi, J., Labonnote, L. C., Goloub, P., Cairns, B., Deuze, J. L., and Tanre, D.: Aerosol Remote Sensing over Clouds Using A-Train Observations, J. Atmos. Sci., 66, 2468-2480, doi:10.1175/2009jas3026.1, 2009b. 